\title{
Human error in strabismus surgery: quantification with a sensitivity analysis
}

\author{
Sander Schutte • Jan Roelof Polling • \\ Frans C. T. van der Helm • Huib J. Simonsz
}

Received: 21 July 2008 /Revised: 22 September 2008 /Accepted: 24 September 2008 / Published online: 25 October 2008

(C) The Author(s) 2008. This article is published with open access at Springerlink.com

\begin{abstract}
Background Reoperations are frequently necessary in strabismus surgery. The goal of this study was to analyze human-error related factors that introduce variability in the results of strabismus surgery in a systematic fashion.

Methods We identified the primary factors that influence the outcome of strabismus surgery. For each of the human-error related factors we quantified variation with clinical assessments: measurement of the angle of strabismus, surgical strategy and surgical accuracy. Firstly, six patients were examined by six orthoptists, and accuracy of prism cover tests was assessed. Secondly, a questionnaire with sample cases $\left(10^{\circ}, 15^{\circ}\right.$ and $20^{\circ}$ of infantile esotropia) was put to orthoptists, to determine variation in current surgical strategy. Finally, photographs made during surgery were analyzed to assess surgical accuracy. The influence of human-error related factors was related to the influence of inter-patient differences with a mechanical model. The relative contribution of all factors was assessed with a sensitivity analysis, and results were compared to clinical studies.

Results The surgical trajectory of strabismus surgery could be modeled mathematically. Measurement of angle of
\end{abstract}

S. Schutte $\cdot$ F. C. T. van der Helm

Department of BioMechanical Engineering,

Faculty of Mechanical, Maritime and Materials Engineering,

Delft University of Technology,

Delft, The Netherlands

J. R. Polling $\cdot$ H. J. Simonsz

Department of Ophthalmology, Erasmus Medical Centre,

Rotterdam, The Netherlands

S. Schutte $(\bowtie)$

Mekelweg 2,

2628CD Delft, The Netherlands

e-mail: mail@schutte.ws strabismus, surgical technique, anatomy and physiology were considered. Variations in the human-error related factors were: (1) the latent angle at distant fixation was measured with a $90 \%$ confidence interval of $5^{\circ}$, and comprised $20 \%$ of the total variance of the postoperative angle, (2) orthoptists decided for bilateral recessions of, respectively, $7.3 \pm 1.7 \mathrm{~mm}$ (total amount of two recessions), $9.1 \pm 1.2 \mathrm{~mm}$ and $10.3 \pm 1.3 \mathrm{~mm}$, which comprised $15 \%$ of the total variance, and (3) surgical accuracy was estimated at $\pm 0.5 \mathrm{~mm}$, which comprised $20 \%$ of the total variance. Conclusion The human error in strabismus surgery could be quantified with a sensitivity analysis. Approximately half of the reoperations in strabismus surgery are caused by inaccuracy in the measurement of the angle of strabismus, variability in surgical strategy and imprecise surgery.

Keywords Strabismus · Strabismus surgery Pediatric ophthalmology Sensitivity analysis . Human error

\section{Introduction}

Strabismus occurs in approximately 4 percent of the population $[1,2]$ and is usually corrected by surgery. In the Netherlands, this is done approximately 150 times per week. Most of these operations are corrections of horizontal eye position by relocating the insertion of one eye muscle on the eye a few millimeters backwards (recession) and resecting the tendon of its antagonist (resection).

The treatment goals for strabismus surgery in adult patients are to alleviate double vision (diplopia) and to improve cosmesis. In children, the treatment goals are to preserve binocular vision in worsening or recent-onset strabismus and to improve cosmesis. Binocular vision 
may be preserved if the eyes are aligned, which can result in better stereopsis [3]. In general, the practical goal of strabismus surgery is to straighten the eyes within $5^{\circ}$ of perfect alignment [4]. This, however, is often not achieved. In a recent study among children with infantile esotropia [5], Polling et al. found that the angle of strabismus was reduced by 1.44 degrees per millimeter of eye-muscle relocation (either recession or resection), with a standard deviation of 0.41 , however. This means the coefficient of variation was $29 \%$ in that study. The large variance in the effect of surgery indicates that the outcome of surgery is unpredictable to some extent.

The variance in the effect of surgery results in reoperations (Fig. 1); patients who are overcorrected or severely undercorrected are usually operated on a second time. The re-operation rate for infantile esotropia, a common type of strabismus, is approximately $20 \%$ [3] when the child is first operated at the age of four, but may be higher when the child is first operated at an earlier age. The proportion of reoperations primarily depends on the variance of the postoperative angle of strabismus. As the chance of diplopia is larger after an overcorrection, patients are systematically undercorrected.

From a process engineering point of view, the variance in the effect of surgery is caused by a number of error

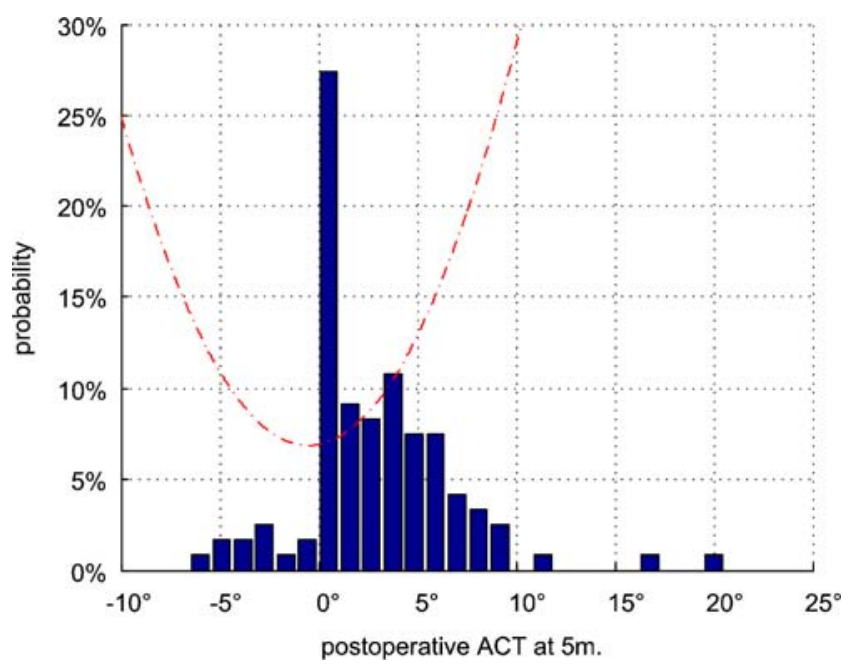

Fig. 1 The empirical probability density of the postoperative angle of strabismus $(N=112)$ as was found in a study comparing bilateral recession (BR) with recession resection (RR) in infantile esotropia [5]. The red line shows the chance of a reoperation given the postoperative angle of strabismus, as was found in a retrospective study [15]. In this study, all patients that were operated over a period of 1 year, in a number of clinics, were contacted to assess their reoperations. If the variability of the postoperative angles is reduced, the number of reoperations decreases. Angles smaller than $-3^{\circ}$ and larger than $6^{\circ}$ are responsible for over $50 \%$ of the reoperations. As the chance of diplopia is larger after an overcorrection, patients are systematically undercorrected sources in the surgical process. The variance propagates through the surgical process and finally becomes visible in the postoperative angle of strabismus. Additional variance is introduced by the fact that patients may respond differently to the same operation because of differences in anatomy and physiology of eye muscles or orbit.

In the preoperative assessment, the patient's angle of strabismus is measured in various directions of gaze. The horizontal, latent angle of strabismus (measured in gaze ahead and at distant fixation) is usually regarded as the most important in deciding on what eye muscles to operate on. The measurements by orthoptists are subject to intrapatient variation, variation of the angle of strabismus during the day, and to inter- and intra-observer variation. The latter two consist of systematic and random errors.

After the preoperative orthoptic assessment, a surgical plan is formulated which consists of a decision for the eye muscles to be operated upon, the surgical technique to be used and the amount of muscle displacement that is needed. The surgical plan is based primarily upon the measured angles of strabismus. However, other non-deterministic factors play a role, such as duration or nature of the strabismus [6]. The goal of the surgical plan is to achieve the optimal postoperative result for individual patients. However, the surgical plan may be subject to variance in the surgical approach or in the planned amount of recession and/or resection. Surgeons' approaches may vary, but also within one surgeon, an approach might be adjusted to individual patients.

Accuracy of the surgical technique is limited, as the actual amount of muscle displacement may well be different from the intended amount. This can be caused by measurement errors, variance in placement and tightening of the sutures, and sagging of muscle tendon between the two points of attachment.

The change in the angle of strabismus by a given relocation of the eye muscle insertions may vary between patients, depending on anatomy and physiological properties. Eye muscle stiffness, for instance, varies between patients, and is usually not taken into account when making the surgical plan. This factor therefore causes variability in the surgical outcome. It is unknown, however, to what extent, in the recovery phase after surgery, amorphous connective tissue develops [7] because tissue is damaged during surgery. It is insufficiently known how wound healing of the eye muscles after strabismus surgery affects their mechanical properties. Possibly also the number of sarcomeres, the contractile element of the muscles, is reduced after a recession operation to adapt to the shorter muscle length [8-10]. Both effects are likely to be subject to inter-patient variation.

Finally, the result of surgery is influenced positively by binocular vision. If a patient has good binocular vision, he 
is able to correct a small strabismus that may remain after surgery. Conversely, binocular vision is only possible if the eyes are aligned. In the randomized controlled trial of Polling et al. [5] among children without binocular vision, a third had gross binocular vision after surgery. These children had better ocular alignment, which may have been either its cause or its consequence. As the exact influence of binocular vision has not yet been quantified, we restricted our analysis to the large group of patients with little or no binocular vision.

To investigate how we can improve the effectiveness of strabismus surgery, we have to study the sources of variability in the surgical trajectory and investigate their influence on the outcome of surgery. If the most influential source of variability, for instance, is surgical accuracy, devices are needed to increase surgical accuracy. If, on the other hand, muscle stiffness is shown to be an important determinant for the outcome of surgery, this should be measured and taken into account when deciding on the surgical plan. The goal of this study was quantification of the human-error related factors in strabismus surgery.

\section{Materials and methods}

We determined the relative contribution of error sources to variability in the effect of strabismus surgery. In a focal group, an assessment was made of all error sources present in strabismus surgery. To relate the error sources to the postoperative angle of strabismus, a mathematical model of the process of strabismus surgery was derived. We carried out a sensitivity analysis on this model to estimate the fraction of reoperations caused by each of the error sources.

The model required input data for each of the error sources. Three of the error sources that were related to human error were studied in detail in clinical assessments. Firstly, the variance in the measurement of the angle of strabismus with the prism cover test was determined. Secondly, the variance in the selection of the surgical plan was assessed with a questionnaire. Thirdly, the accuracy of relocating the eye muscles during surgery was estimated. Finally, a comparison was made with clinical studies in order to validate the model.

Clinical assessment of the variance in the measurement of the angle of strabismus

We determined whether orthoptists can measure the angle of strabismus in a patient with sufficient accuracy. Six orthoptists from three university eye clinics were asked to measure angles of strabismus in six patients. All orthoptists had more than 5 years of experience in three of the academic clinics of the Netherlands. Oral informed consent was obtained from the participating patients. The patients were examined by all orthoptists in a predefined, randomized, order. The orthoptists were not able to exchange information in any way. None of the patients had been examined by any of the participating orthoptists before. Only general information about the patient was supplied: date of birth, glasses worn, visual acuity, refractive error, previous strabismus surgery and the use of medication. Horizontal and vertical, latent and manifest angles of strabismus were measured during fixation at near and at distance fixation using prism cover tests.

Accuracy of the measurements was quantified as standard deviation of the measurements of the orthoptists for each type of measurement. The standard deviations of the four types of measurements of horizontal and vertical angles were compared with a statistical F-test [11]. We used a one-tailed test with a significance level of $5 \%$. To be able to perform this test on all measurements of one type (e.g., the manifest angle in distant fixation) the 36 measurements of one type were grouped. To compare variance between measured angles, the average of the six measurements of one type of measurement on a patient was subtracted from every measurement on that patient such that the mean of all measurements became zero. Next, standard deviations of the groups were compared with the other groups. We compared all four types of horizontal measurements to all four types of vertical measurements. Within the horizontal and vertical measurements, we compared every type of measurement to the three other types of measurements.

\section{Clinical assessment of the variance in surgical strategy}

The measured angle of strabismus is the main determinant in deciding on the surgical plan. We investigated variability of the surgical strategy by distributing a questionnaire during the annual meeting of the society for Dutch strabismologists and orthoptists. The questionnaire described three sample patients with infantile esotropia. Each sample patient was a 3 -year-old with infantile esotropia without binocular vision and with average glasses $(S+1 / S+1)$. Case 1 had an angle of strabismus of $10^{\circ}$ (measured with an alternating prism cover test at $5 \mathrm{~m}$ ). Case $2 \mathrm{had}$ an angle of strabismus of $15^{\circ}$. Case 3 had an angle of strabismus of $20^{\circ}$.

Orthoptists were asked to decide on the distance of relocation of the eye muscles for each of the patients. After 10 minutes the completed questionnaires were collected, under strict supervision.

The main outcome parameter was the variance in the prescribed amount of surgery in millimeters of surgery for either bilateral recession (BR) or recession resection (RR). Note that this is the variance in the decision-making process, because all orthoptists had the same data available. Part of the variability is caused, however, by adaptation to 
the systematic errors during surgery by a particular surgeon. Differences in total amount of prescribed surgery between $\mathrm{BR}$ and RR were analyzed with the Student's $t$-test, with a significance level of $5 \%$.

\section{Clinical assessment of the variance in surgical accuracy}

During strabismus surgery, the insertions of the eye muscles on the eye are altered to change the angle of strabismus in different directions of gaze. Surgical accuracy or mechanical differences between patients' eye muscles influence the postoperative result of surgery. Surgical accuracy was estimated based upon the photographs from the Bilateral Recession vs Recession Resection Study [5]. In this study, photos were taken during two stages of the operation in either recession or resection: (1) after fitting the sutures through the muscle, and (2) before closing the conjunctiva. A millimeter ruler next to the muscle was photographed with the eye. During the recession, four points were marked with methylene blue on the sclera: two points next to the suture knots in the muscle at the insertion, and two points posterior on the sclera at the planned distance of recession on either side of the muscle. During the resection, four points were marked with methylene blue on the sclera: two points at the muscle insertion on the sclera, and two points posterior from these two on the muscle at the planned distance of recession of the muscle. In resection, the muscle was sutured at the level of these two posterior points, clamped in a Bangerter myostat and cut off from the insertion. If the central part of the resected or recessed muscle hung between the sutures, a third suture was placed to get the central part of the muscle in line. We estimated the maximum achievable accuracy based upon the photographs.

Mathematical model of the surgical trajectory

To obtain a comprehensive insight into the contributions of the three human-error related factors described above in the surgical trajectory, we derived a mathematical model of the surgical treatment trajectory for horizontal strabismus. The postoperative angle was expressed as a function of the measured preoperative angle of strabismus, surgical dosage, amount of surgery and anatomical and physiological parameters.

To compare the variance caused by human-error related factors to the variance caused by inter-patient differences in anatomy and physiology, we used a straightforward mechanical model (Appendix A). The mechanical model enabled estimation of the effect per millimeter of surgery for different values of anatomical and physiological parameters. The model has 1 degree of freedom (horizontal eye rotation), and only two eye muscles are present. The model comprised eye radius ( $\mathrm{r}[\mathrm{mm}])$, linearized muscle stiffness $(\mathrm{k}[\mathrm{N} / \mathrm{mm}])$ and linearized stiffness in passive rotation $(\mathrm{g}[\mathrm{mNm} / \mathrm{rad}])$.

A sensitivity analysis was carried out to investigate how much influence each of the error sources has on the postoperative result of strabismus surgery. Sensitivity was analytically derived and numerical evaluations were performed. The sensitivity analysis resulted in variance of the postoperative angle of strabismus for each error source.

The sensitivity analysis enabled evaluation of the variability of the postoperative angle of strabismus for a group of patients with a normal distribution of the parameters. For estimating sensitivity, the mean value and variance of each parameter were required. For preoperative measurement accuracy, we used the variance found in our clinical assessment. Mean and standard deviation of the eye radius [12] and muscle stiffness [13] were obtained from literature data. Some course measurements [14], of stiffness in passive rotation were used in the sensitivity analysis. Result of this evaluation was the variance (in degrees) of the postoperative angle of strabismus for each error source.

If a patient has good binocular vision, he is able to correct a small strabismus that may remain after surgery. Accordingly, the result of strabismus surgery is better and more stable, when binocular vision is good. This effect was neglected in our model.

To validate the estimates of the sensitivity analysis, we compared the total percentage of reoperations to literature data.

\section{Results}

Error sources in the surgical trajectory of strabismus surgery were mapped out (Fig. 2). The trajectory starts with the preoperative measurements of the patient. Based upon these measurements, a surgical plan is determined. Surgery follows and after the recovery phase, a postoperative assessment is carried out.

We mathematically modeled the processes in the surgical trajectory, and carried out a sensitivity analysis on this model to estimate the fraction of reoperations caused by each of the error sources. The input data for the model were acquired from the literature and from three clinical assessments of variance introduced by human error.

Clinical assessment of the variance in the measurement of the angle of strabismus

Age and diagnoses of the six patients are shown in Table 1. All six patients gave their informed consent prior to participating in this study. All patients were examined by all orthoptists, and an examination sheet was returned for every patient. Manifest and latent vertical angles of 
Fig. 2 A schematic representation of the surgical trajectory of strabismus. Error sources that influence the outcome of surgery are depicted in the middle column. The error sources related to human error are marked red. The right column shows the nomenclature of the parameters in the mathematical model

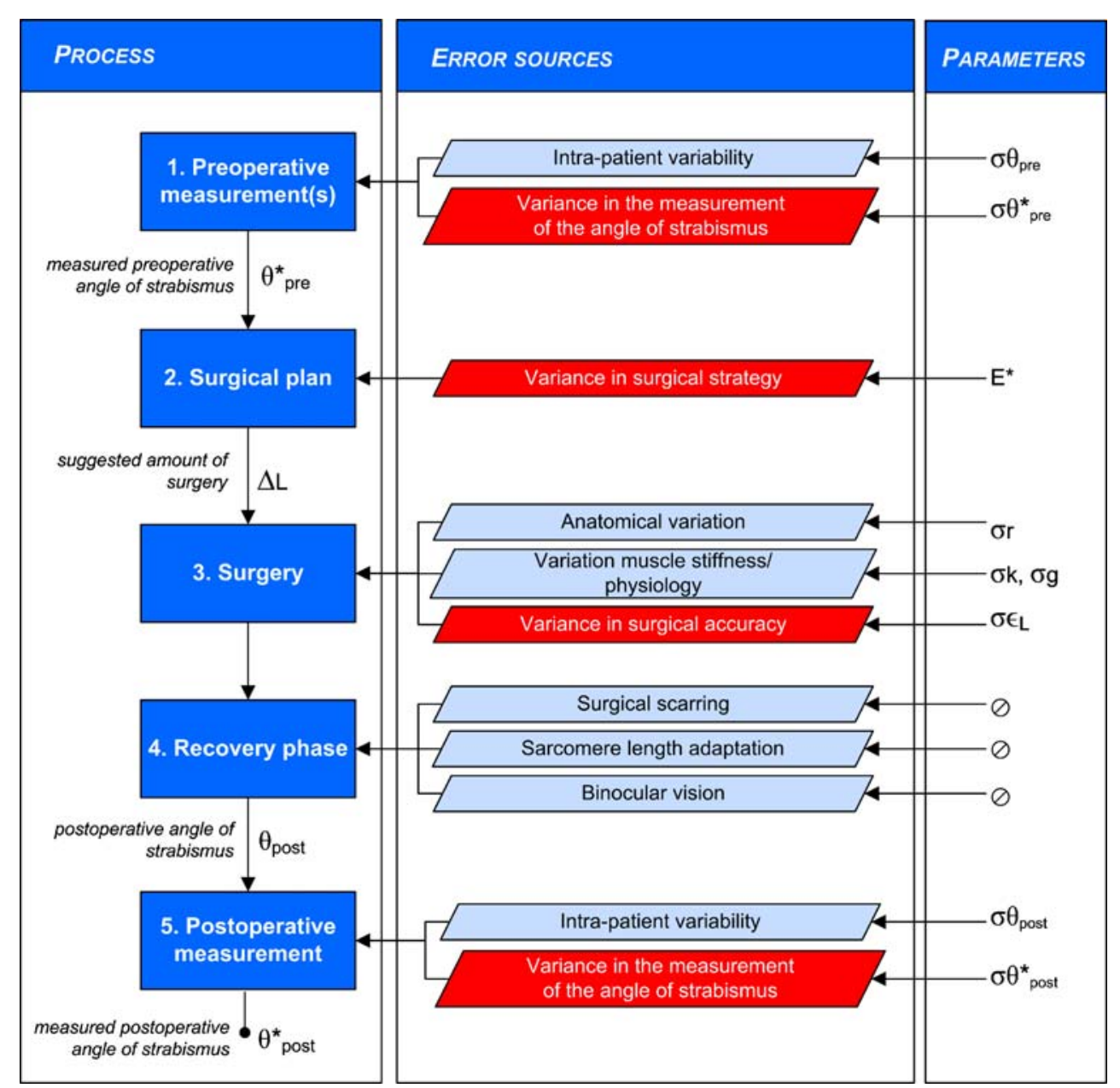

strabismus, at distance and at near fixation, were measured more accurately than their corresponding manifest and latent horizontal angles of strabismus, at distance and near fixation (Table 2). Horizontal manifest and latent angles were measured more accurately at distant fixation than at near fixation. Horizontal latent angles were measured more accurately than horizontal manifest angles. Especially the angles at near fixation showed a large variation (Fig. 3). At distance fixation, horizontal latent angles were measured more accurately than horizontal manifest angles. The horizontal angle of strabismus that could be assessed most reliably was the latent angle of strabismus at distance fixation, with an average standard deviation of $1.72 \pm 0.45 \mathrm{deg}$.

Table 1 Age and diagnosis of the six patients that participated in the clinical assessment of the variance in the measurement of the angle of strabismus
Clinical assessment of the variance in surgical strategy

One hundred and eighty four Dutch orthoptists filled out the questionnaire with three example cases of strabismus, in complete silence. Four orthoptists did not use the prescribed notation protocol, and were excluded from the analysis. Twelve percent of the orthoptists decided not to operate on the patient with $10^{\circ}$ of esotropia at all. In all three patients, most of the orthoptists favored BR over RR. With a larger angle of strabismus, more orthoptists prescribed BR with a 'loop' $(0 \%, 4 \%$ and $28 \%$ respectively). In bilateral recession, the orthoptists prescribed $7.3 \pm 1.7 \mathrm{~mm}, 9.1 \pm$ $1.2 \mathrm{~mm}$ and $10.3 \pm 1.3 \mathrm{~mm}$ (mean $\pm \mathrm{SD}$ ) muscle relocation for $10^{\circ}, 15^{\circ}$ and $20^{\circ}$ of esotropia (Fig. 4). In the patient

\begin{tabular}{lll}
\hline Patient & Age (years) & Diagnosis \\
\hline 1 & 7 & Infantile esotropia, dissociated vertical deviation and upshoot in adduction \\
2 & 8 & Consecutive exotropia, A pattern and high AC/A ratio \\
3 & 9 & Exophoria \\
4 & 14 & Intermittent exotropia, upshoot in adduction and V pattern \\
5 & 76 & Esotropia with V pattern \\
6 & 79 & Consecutive exotropia with right hypertropia and V pattern \\
\hline
\end{tabular}


Table 2 The mean standard deviations of the examinations and their standard deviations

\begin{tabular}{|c|c|c|c|c|c|c|c|}
\hline & \multicolumn{3}{|c|}{ Distant fixation } & & \multicolumn{3}{|l|}{ Near fixation } \\
\hline & Manifest & & Latent & & Manifest & & Latent \\
\hline Horizontal & $\left(4.19 \pm 2.84^{\circ}\right.$ & $>$ & $\left.1.74 \pm 0.45^{\circ}\right)$ & $<$ & $\left(5.44 \pm 3.07^{\circ}\right.$ & $>$ & $\left.3.97 \pm 1.33^{\circ}\right)$ \\
\hline Vertical & $\left(1.75 \pm 0.63^{\circ}\right.$ & $\circ$ & $\left.1.42 \pm 0.57^{\circ}\right)$ & $\circ$ & $\left(1.21 \pm 1.02^{\circ}\right.$ & ○ & $\left.1.45 \pm 0.96^{\circ}\right)$ \\
\hline
\end{tabular}

All measurements were performed six times by different orthoptists in six patients. Significant differences are denoted by' $<$ ' or ' $>$ '; a nonsignificant difference is denoted by ' $O$ '. Horizontal angles are measured less accurately than vertical angles. Horizontal angles of strabismus measured at distance fixation are measured more accurately than at near fixation. Horizontal latent angles are measured more accurately at near and at distance fixation. The most reliable horizontal angle is the latent angle of strabismus measured at distance fixation. There was no significant difference between the four types of vertical measurements.

with $20^{\circ}$ of esotropia, orthoptists who suggested performing RR decided on a significantly higher dose than the orthoptists who chose BR. In the patients with $10^{\circ}$ and $15^{\circ}$ of esotropia, these differences were not significant.

Clinical assessment of the variance in surgical accuracy

Deviations from the intended amount of surgery occur in measurements, in reattaching the muscle to the sclera and by sagging of the muscle between the attachment points. We analyzed 30 cases, and estimated surgical accuracy at $0 \pm$ $0.5 \mathrm{~mm}$ (mean $\pm \mathrm{SD})$.

Fig. 3 A graphical representation of all measurements that were performed. Each frame represents measurements on one patient $(N=6)$; each box plot represents all measurements $(N=6)$ of one type of angle of strabismus. The following angles were measured: the manifest angle in distant fixation (DM), the latent angle in distant fixation (DL), the manifest angle in near fixation (NM) and the latent angle in near fixation (NL). Red box plots represent horizontal angles; green box plots vertical angles of strabismus
Mathematical model of the surgical trajectory

A mathematical model of primary horizontal strabismus surgery was derived. The postoperative angle of strabismus $\left(\theta_{\text {post }}\right)$ was expressed as the preoperative angle of strabismus $\left(\theta_{\text {pre }}\right)$ minus the amount of surgery $(\Delta L)$ multiplied by the actual effect of surgery $(E)$ :

$\theta_{\text {post }}=\theta_{\text {pre }}-\Delta L \cdot E$

To obtain the prescribed amount of surgery $(\Delta L)$, the measured preoperative angle of strabismus $\left(\theta^{*}{ }_{\text {pre }}\right)$ is divided by the expected effect of surgery $\left(E^{*}\right)$. The error
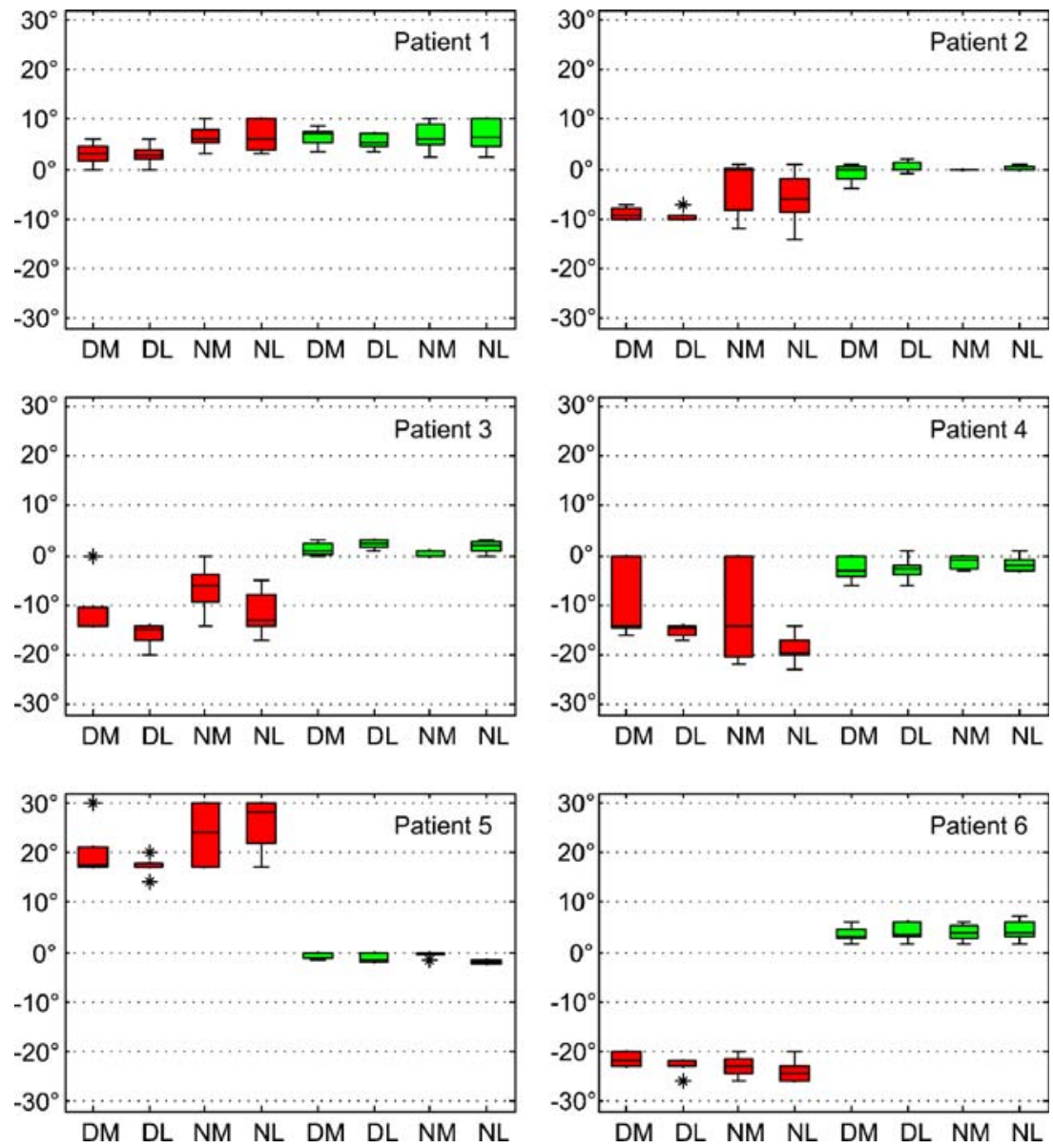


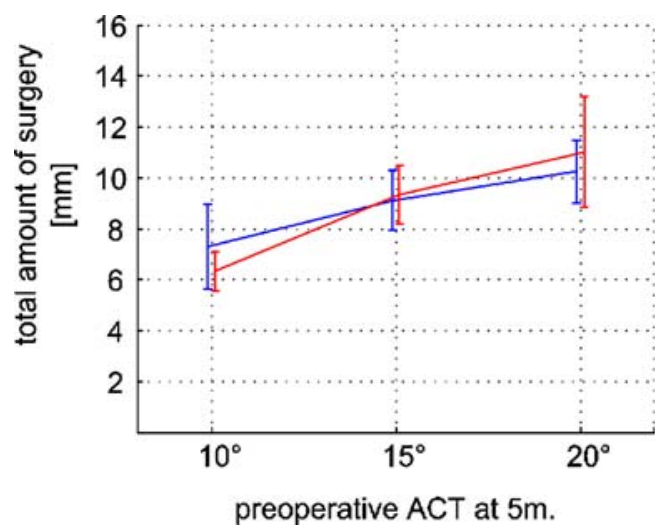

Fig. 4 A graphical representation of the outcome of the questionnaire. The horizontal axis represents the preoperative angle of strabismus. The vertical axis depicts the total amount of prescribed surgery (mean $\pm \mathrm{SD}$ ) for bilateral recession (BR) in red and for recession resection (RR) in blue

made during surgery $(\varepsilon L)$ is added to this intended amount of surgery:

$\Delta L=\left(\frac{\theta_{p r e}^{*}}{E^{*}}+\varepsilon L\right)$

The theoretical effect of surgery $(E)$ was derived with a straightforward mechanical model (Appendix A). The mechanical model comprises eye radius $(r)$, muscle stiffness $(k)$ and stiffness in passive rotation $(g)$. The effect of surgery can be expressed as:

$E=\frac{k r}{\left(2 k r^{2}+g\right)}$

Substitution of Eq. 3 and Eq. 2 in Eq. 1 finally yields the postoperative angle of strabismus as a function of all of the previously described parameters:

$\theta_{\text {post }}=\theta_{\text {pre }}-\left(\frac{\theta_{\text {pre }}^{*}}{E^{*}}+\varepsilon L\right) \cdot \frac{k r}{\left(2 k r^{2}+g\right)}$

Summarizing, the model derived in Eq. 4 provides the postoperative angle of strabismus as a function of the actual and measured preoperative angles of strabismus, the expected dose response ratio, the error made during surgery, and the patient's mechanical characteristics: muscle stiffness, eye radius, and stiffness in passive rotation.

To make estimates of variance within the surgical trajectory, we carried out sensitivity analysis of the model. If we would substitute mean values of a population of strabismus patients into Eq. 4, we would estimate the mean postoperative angle of strabismus for that population. In addition, the model can be used to estimate how much each error source contributes to variance in the postoperative angle of strabismus if we perform a sensitivity analysis. The delta method [16], a statistical method, can be used to approximate the variance of a function of one or more variables. If we assume the parameters are independent, variance (squared standard deviation) in the postoperative angle can be written as:

$$
\begin{aligned}
\sigma_{\theta_{\text {post }}}^{2} & \approx\left(\frac{\partial \theta_{\text {post }}}{\partial \theta_{\text {pre }}}\right)^{2} \sigma_{\theta_{\text {pre }}}^{2}+\left(\frac{\partial \theta_{\text {post }}}{\partial \theta_{\text {pre }}^{*}}\right)^{2} \sigma_{\theta_{\text {pre }}}^{2}+\left(\frac{\partial \theta_{\text {post }}}{\partial E^{*}}\right)^{2} \sigma_{E^{*}}^{2} \\
+ & \left(\frac{\partial \theta_{\text {post }}}{\partial \varepsilon L}\right)^{2} \sigma_{\varepsilon L}^{2}+\left(\frac{\partial \theta_{\text {post }}}{\partial r}\right)^{2} \sigma_{r}^{2}+\left(\frac{\partial \theta_{\text {post }}}{\partial k}\right)^{2} \sigma_{k}^{2} \\
& +\left(\frac{\partial \theta_{\text {post }}}{\partial g}\right)^{2} \sigma_{g}^{2}
\end{aligned}
$$

In which the partial derivatives of Eq. 4 represent the sensitivities, based upon average values of the parameters. Multiplication with variance of each parameter then provides the estimated contributions to variance in the postoperative angle of strabismus. Influence of the individual error sources was quantified by evaluating the individual terms of Eq. 5 .

In order to evaluate Eq. 5 numerically, the mean and variance are required for each parameter. The numerical values that were used are summarized in Table 3 . We estimated the SD of intra-patient variation of the angle of strabismus $\left(\theta_{\text {pre }}\right)$ at \pm 1 degree. For the measurement accuracy of the preoperative angle of strabismus $\left(\theta^{*}{ }_{p r e}\right)$ we assumed the mean measurement value to be the actual angle of strabismus. For the SD we used the deviation found in the latent angle with distant fixation $\left(1.72^{\circ}\right)$, as this angle is used most often for making the surgical plan. We assumed the orthoptic measurements are repeated three times before surgery. The standard deviation then becomes $1^{\circ}\left(1.72 / \sqrt{3} \approx 1^{\circ}\right)$. For the surgical strategy $\left(E^{*}\right)$, or the expected dose-response ratio, we used the values as found with our questionnaire: $1.4 \pm 0.17,1.65 \pm 0.08,1.94 \pm$ $0.07 \% / \mathrm{mm}$ for preoperative angles of $10^{\circ}, 15^{\circ}, 20^{\circ}$ respectively. For the surgical accuracy $(\varepsilon L)$ we used the variance as found in the clinical assessment, $\pm 0.5 \mathrm{~mm}$, and we expected the mean deviation from the intended amount of surgery to be zero, i.e. we assumed that there are no systematic errors. Usually, two muscles are operated in one patient so the surgical error is made twice. Variances (squared SD) can be added, and the total standard deviation becomes $\sqrt{2 \times 0.5^{2} \mathrm{~mm}} \approx 0.7 \mathrm{~mm}$.

To model influence of the variance of anatomical and physiological factors, variances were derived for the size of the eye, for muscle stiffness and for stiffness in passive rotation of the eye. We used an eye radius $(r)$ of $11.6 \pm$ $0.5 \mathrm{~mm}$ [12]. Muscle stiffness $(k)$ has previously been measured under local anesthesia [13], and was found to be $35 \pm 6.1 \mathrm{~N} / \mathrm{m}$. Two attempts have been made to assess the stiffness in passive rotation of the human eye $(g)$ in vivo $[17,18]$. In both measurements, displacement of the eye in its orbit was neglected [19]. These are the only in-vivo 
Table 3 Mean values and standard deviations that were used to estimate the variability caused by each error source

\begin{tabular}{|c|c|c|c|c|}
\hline \multicolumn{2}{|l|}{ Parameter } & \multirow{2}{*}{$\begin{array}{l}\text { Mean } \\
10^{\circ}, 15^{\circ}, 20^{\circ}\end{array}$} & \multicolumn{2}{|l|}{ SD } \\
\hline Preoperative angle of strabismus & $\theta_{\text {pre }}$ & & $1^{\circ}$ & \\
\hline Measured preoperative angle of strabismus & $\theta_{\text {pre }}^{*}$ & $10^{\circ}, 15^{\circ}, 20^{\circ}$ & $1^{\circ}$ & \\
\hline Surgical strategy & $E^{*}$ & $1.4,1.65,1.94$ & $0.17,0.08,0.07$ & $\% / \mathrm{mm}$ \\
\hline Surgical accuracy & $\varepsilon L$ & 0 & 0.7 & $\mathrm{~mm}$ \\
\hline Eye radius & $r$ & 11.6 & 0.5 & $\mathrm{~mm}$ \\
\hline Muscle stiffness & $k$ & 35 & 6.1 & $\mathrm{~N} / \mathrm{m}$ \\
\hline Stiffness in passive rotation & $g$ & 6 & 0.9 & $\mathrm{mNm} / \mathrm{rad}$ \\
\hline
\end{tabular}

measurements to date, however, and we used the measurement values from these studies $(6 \pm 0.9 \mathrm{mNm} / \mathrm{rad})$.

After substitution of numerical values, the sensitivity analysis predicted a total standard deviation of approximately $5.5^{\circ}$ (Fig. 5). In our mathematical model, we assumed that intra-patient variability of the angle of strabismus was the same postoperatively, and causes approximately $18 \%$ of the total variance. The variability in the preoperative measurement caused $14 \%, 17 \%$ and $18 \%$ of the total variance for preoperative angles of $10^{\circ}, 15^{\circ}$ and $20^{\circ}$ respectively. The variance in surgical strategy caused $22 \%, 12 \%$ and $10 \%$ respectively. The error in recession/resection $( \pm 0.5 \mathrm{~mm})$ caused approximately $20 \%$ of the variance. The inter-patient differences in anatomical and physiological factors together caused approximately $25 \%, 33 \%$ and $38 \%$ respectively. The most influential parameter was muscle stiffness. Eye radius played only a minor role.

The total human-error related factors, i.e. surgical accuracy and accuracy in measurement of the angle of strabismus, cause approximately $50 \%$ of the variability. The remaining $50 \%$ is caused by anatomical and physiological variation and by day-to-day variability of the patient.

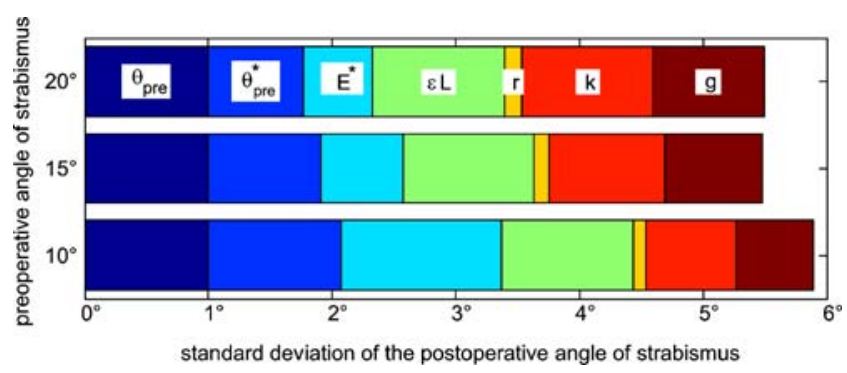

Fig. 5 Estimated contributions to the standard deviation of the postoperative angle of strabismus surgery for intra-patient variation ( $\theta$ pre $)$, inter-observer variation $\left(\theta^{*}\right.$ pre $)$, variation in surgical strategy $\left(E^{*}\right)$, surgical inaccuracy $(\varepsilon L)$, variance in eye radius $(r)$, variance in muscle stiffness $(k)$ and variance in stiffness in passive rotation $(g)$. These estimates are based upon the mean values and standard deviations provided in Table 3

\section{Discussion}

The human error in strabismus surgery could be quantified with a sensitivity analysis. The systematic approach led to a model of the processes constituting the trajectory of strabismus surgery, in which the relative contribution to variability of the surgical result could be estimated for several human-error related factors.

In many medical treatments, the outcome is often not satisfactory and the number of error sources that influence the outcome is large. In order to make improvements in these treatments, it should be known how much influence each of the error sources has on the treatment outcome. The approach that we have presented proved to be useful for strabismus surgery, but might also be valuable for other medical treatments. Firstly, the treatment trajectory has to be analyzed systematically to map out all processes and error sources; secondly a mathematical model has to be derived; and finally, a sensitivity analysis has to be performed to investigate the relative importance of the variables. This technique provides new insights, and shows where improvements in the trajectory have the largest effect. The input data that is required to perform a sensitivity analysis might be available in literature; otherwise clinical assessments are needed to obtain these data.

The standard deviation of the postoperative angle of strabismus that we predicted based upon the sensitivity analysis $\left(5.5^{\circ}\right)$ was comparable to the standard deviation found in a randomized clinical trial $\left(4.8^{\circ}\right)$ in which bilateral recession was compared to recession resection surgery [5]. We found that human errors caused approximately half of the variability of the postoperative angle of strabismus. Hence, they cause approximately half of the reoperations.

The firstly identified human factor in the surgical trajectory was the measurement of the angle of strabismus. Accuracy of the measurement of the angle of strabismus with prism cover tests limits the final result. The measurement of the horizontal manifest angle of strabismus measured with fixation at near was highly unreliable, with an average standard deviation of $5.44 \pm 3.07^{\circ}$. This might be because of different accommodative efforts, and because the influence 


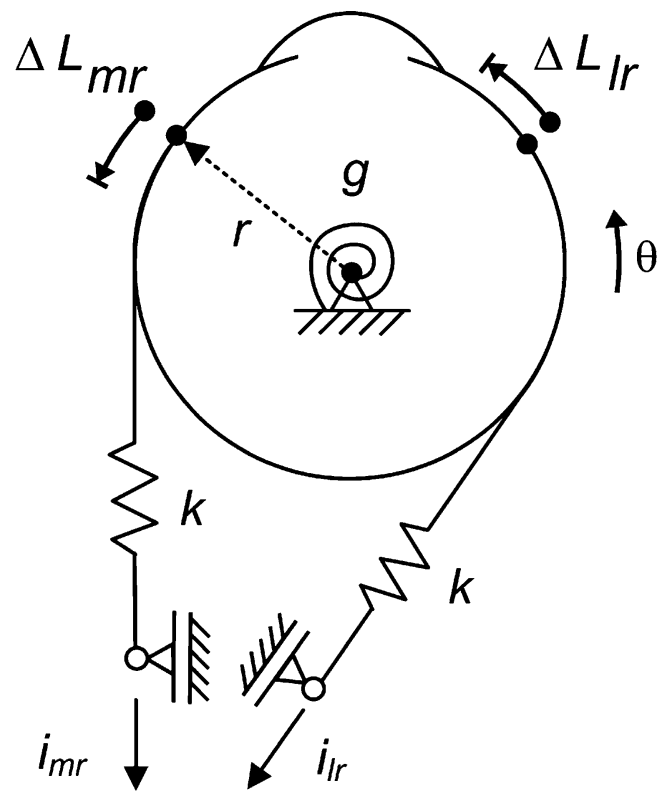

Fig. 6 A schematic representation of the mechanical model that was used to derive the influence of surgical inaccuracy. The model comprised eye radius $(r \quad[\mathrm{~mm}])$, linearized muscle stiffness $(k[\mathrm{~N} / \mathrm{mm}])$ and linearized stiffness in passive rotation $(g[\mathrm{mNm} / \mathrm{rad}])$

of varying distances is larger with fixation at near than the influence with fixation at distance. The horizontal angle of strabismus that was measured most accurately was the horizontal angle of strabismus with fixation at distance, with an average standard deviation of $1.72 \pm 0.45^{\circ}$. If three measurements are carried out preoperatively, and the average angle is used for surgical planning, measurement inaccuracy causes one fifth of the overall variability. Measurement variability in the manifest angles of strabismus might be larger than variability in the latent angles because of the influence of binocular vision and because of the more difficult way of measuring manifest angles.

In addition to the measurement variability, the angle of strabismus itself may vary over time. By taking a number of measurements, the estimate of the average angle becomes more accurate. Automated measurements, for instance by employing Purkinje imaging [20], may improve the measurement accuracy and decrease the influence of this error.

The second human factor in the surgical trajectory is the surgical strategy, primarily based upon the dose-response relationship. We found that the variability of the prescribed amount of surgery is relatively large. The variability in the surgical strategy causes approx. one fifth of the overall variance in the postoperative angle for patients with a preoperative angle of $10^{\circ}$. Part of this variability may reflect a compensation for systematic errors made, for instance in the measurement of the angle of strabismus or during surgery: if the recessions of a particular surgeon are systematically slightly larger than those of other surgeons, the orthoptists may, consciously or not, compensate for that systematic error by systematically suggesting less muscle displacement during surgery.

It is important to realize that the surgical plan is not equal to the expected average effect. Polling et al. [5] found $1.44 \% \mathrm{~mm}$ for recession resection surgery, whereas most guidelines $[10,21-26]$ and our assessment amongst Dutch orthoptists employ higher dose-response ratios $\left(1.4-1.9^{\circ} / \mathrm{mm}\right)$. This discrepancy is the result of a systematic undercorrection, because an overcorrection of strabismus is more likely to cause diplopia and loss of binocular vision. There are a large number of guidelines in the literature for the amount of recession/resection per angle of strabismus. The guidelines vary, and there is little agreement. Validated guidelines, if broadly adopted, would decrease the variability and reduce the number of reoperations.

The effect of surgery is usually expressed in $\% / \mathrm{mm}$, and is calculated by subtracting the preoperative angle from the postoperative angle and dividing by the total amount of muscle surgery. In some of the studies about the doseresponse relation in strabismus surgery, the effect of surgery is related to the preoperative angle. This should be discouraged. Because the preoperative angle of strabismus is used to calculate the effect of surgery, measurement errors in the preoperative angle of strabismus cause an artifact: the effect of surgery seems larger for larger preoperative angles, and smaller for smaller preoperative angles of strabismus, as compared to the actual effect.

Surgical accuracy proved to be the third important human factor in the surgical trajectory. Surgical accuracy probably varies between individual surgeons [27]. It is, however, unlikely that the accuracy is better than $\pm 0.5 \mathrm{~mm}$. Based upon this accuracy, we estimated that approximately one fifth of the reoperations are caused by imprecise surgery. New surgical instruments or use of adhesives [28] might reduce this variability.

Adjustable sutures [29] are used in an effort to overcome the influence of variability in strabismus surgery. During surgery the muscle tendon is secured with sutures that can be adjusted, either with the 'bow tie' or with the 'sliding knot' technique. When the patient is sufficiently alert after surgery, adjustments can be made to the muscle relocation under local anesthesia. A disadvantage is that it requires considerable cooperation from the patient, and therefore is not usable in young patients. A recent Cochrane review [30] concluded that it could not be concluded from current literature that adjustable sutures produces a more accurate long-term ocular alignment than conventional surgery.

Finally, the relative contribution of anatomical and physiological variation was assessed, in comparison to human-related variability. With a straightforward mechanical model and literature data, we estimated that up to one third of the overall variability in strabismus surgery could be caused by interindividual variation in anatomy and 
physiology. The stiffness of the muscles and the stiffness of the eye in passive rotation can be assessed with a forced duction test in an effort to reduce variability, especially in reoperations. Our model predicted that the size of the eye plays only a minor role in average patients. This agrees with the clinical data from Kushner et al. [31, 32]. To decrease the influence of anatomy and physiology, new devices should be aimed at measuring the individual mechanical properties of patients and relating these parameters to the effect of surgery. If these relationships are known, the optimal surgical strategy can be determined after individual measurements pre- or intraoperatively.

A weakness of our study was that we neglected the influence of binocular vision. As the exact influence of binocular vision has not yet been quantified, we restricted our analysis to the large group of patients with little or no binocular vision. If binocular vision develops postoperatively, the outcome of surgery is better.

Open Access This article is distributed under the terms of the Creative Commons Attribution Noncommercial License which permits any noncommercial use, distribution, and reproduction in any medium, provided the original author(s) and source are credited.

\section{Appendix A}

The mechanical model for strabismus surgery comprised eye radius, linearized muscle stiffness and linearized stiffness in passive rotation (Fig. 6). The model is based upon the first strabismus model by Robinson [33], as it was assumed that activation to the strabismic eye does not change after surgery.

With regard to muscle stiffness, Robinson [34], Collins [17] and Simonsz [18] found that the relation between length and force of a contracting eye muscle is approximately linear, whether it contracts moderately or strongly. Force development by the muscles is therefore only dependent on elongation and activation in the static gaze. The force in a non-contracting eye muscle during passive elongation rises exponentially with length. In our model, we assumed a pretension in the muscles sufficiently high that passive elongation is never reached. Forces that act on the eye by the medial rectus muscle $\left(F_{m r}\right)$ and the lateral rectus muscle $\left(F_{l r}\right)$ were reduced to linear relationships. In these relationships, stiffness of the muscle $(k)$ is multiplied by its elongation due to activation $\left(i_{m r}\right.$ and $\left.i_{l r}\right)$, rotation of the eye $(\theta)$ in radians times the eye radius $(r)$ and surgery $\left(\Delta L_{m r}\right.$ and $\left.\Delta L_{l r}\right)$ :

$F_{m r}=k\left(i_{m r}-\theta r+\Delta L_{m r}\right)$

$F_{l r}=k\left(i_{l r}+\theta r+\Delta L_{l r}\right)$
Stiffness in passive rotation was modeled as a linear torsional spring. This parameter represents stiffness that is caused by deformation of orbital fat by the muscles and the optic nerve. The passive moment $(M)$ is always counteracting rotation and linearly dependent on torsional stiffness $(g)$ :

$M_{g}=-\theta g$

In any static position of the eye, the sum of moments acting on the eye has to be zero. The moment balance around the center of the eye is given by:

$\Sigma M=r F_{m r}-r F_{l r}+M_{g}=0$

Substitution of Eq. A.1, A.2 and Eq. A.3 in Eq. A.4 and solving for $\theta$ gives the rotation of the eye as a function of surgery and activation:

$\theta=E\left(i_{m r}-i_{l r}\right)+E\left(\Delta L_{m r}-\Delta L_{l r}\right)$

in which:

$E=\frac{k r}{\left(2 k r^{2}+g\right)}$

For strabismus surgery, we assume the angle of rotation is the preoperative angle of strabismus $\left(\theta=\theta_{\text {pre }}\right)$ that has to be compensated. Activation remains the same after surgery, and therefore has no influence on the change in angle of strabismus, and the first term in Eq. A.5 diminishes. If we perform the same amount of surgery on both muscles, $\Delta L_{l r}=$ $-\Delta L_{m r}$, the total amount of surgery for preoperative angle $\theta_{\text {pre }}$ becomes $\Delta \mathrm{L}=2 \Delta \mathrm{L}_{\mathrm{mr}}$, substitution in Eq. A.5 and rewriting gives:

$E=\frac{\theta_{\text {pre }}}{\Delta L}$

In which $(E)$ proves to be the actual effect per amount of surgery, which is defined as the change in angle of strabismus divided by the total amount of surgery. Summarizing, we derived the effect of surgery as a function of muscle stiffness, eye radius and stiffness in passive rotation.

\section{References}

1. Abrahamsson M, Magnusson G, Sjostrand J (1999) Inheritance of strabismus and the gain of using heredity to determine populations at risk of developing strabismus. Acta Ophthalmol Scand 77 (6):653-657, doi:10.1034/j.1600-0420.1999.770609.x

2. Greenberg AE et al (2007) Incidence and types of childhood esotropia: a population-based study. Ophthalmology 114(1):170 174, doi:10.1016/j.ophtha.2006.05.072

3. Simonsz HJ, Kolling GH, Unnebrink K (2005) Final report of the early vs. late infantile strabismus surgery study (ELISSS), a 
controlled, prospective, multicenter study. Strabismus 13(4):169199, doi:10.1080/09273970500416594

4. Donahue SP (2007) Clinical practice. Pediatric strabismus. N Engl J Med 356(10):1040-1047 doi:10.1056/NEJMcp051888

5. Polling $J$ et al (2008) A randomized comparison of bilateral recession with unilateral recession-resection as surgery for infantile esotropia. (submitted for publication)

6. Von Noorden G, Burian H (1985) Burian-von Noorden's Binocular Vision and Ocular Motility: Theory and Management of Strabismus. Vol. 3. Mosby, St. Louis

7. Ludwig IH, Chow AY (2000) Scar remodeling after strabismus surgery. J AAPOS 4(6):326-333, doi:10.1067/mpa.2000.107899

8. Goldspink G et al (1974) Effect of denervation on the adaptation of sarcomere number and muscle extensibility to the functional length of the muscle. J Physiol 236(3):733-742

9. Hayat A et al (1978) Effects of denervation on the reduction of sarcomere number in cat soleus muscle immobilized in shortened position during seven days. J Physiol (Paris) 74(6):563-567

10. Mims JL III, Treff G, Kincaid M (1985) Quantitative surgical guidelines for bimedial recession for infantile esotropia. Binoc Vis $1: 7-22$

11. Snedecor GW, Cochran WG (1989) Statistical Methods, Eighth Edition. Iowa State University Press, Ames

12. Larsen JS (1971) The sagittal growth of the eye. IV. Ultrasonic measurement of the axial length of the eye from birth to puberty. Acta Ophthalmol (Copenh) 49(6):873-886

13. Simonsz HJ (1994) Force-length recording of eye muscles during local anesthesia surgery in 32 strabismus patients. Strabismus 2:197-218, doi:10.3109/09273979409035475

14. Collins CC, Scott AB, O'Meara DM (1969) Elements of the peripheral oculomotor apparatus. Am J Optom Arch Am Acad Optom 46(7):510-515

15. Van de Vijver-Reenalda $H$ et al (1999) Cumulatieve kans op heroperatie gerelateerd aan de postoperatieve scheelzienshoek bij congenitaal scheelzien: een retrospectief onderzoek. Ned Tijdschr Genees, $\mathrm{p} 143$

16. Oehlert GW (1992) A Note on the Delta Method. Am Stat 46 (1):27-29, doi:10.2307/2684406

17. Collins CC et al (1981) Extraocular muscle forces in normal human subjects. Invest Ophthalmol Vis Sci 20(5):652-664

18. Simonsz HJ et al (1986) Intraoperative length and tension curves of human eye muscles. including stiffness in passive horizontal eye movement in awake volunteers. Arch Ophthalmol 104 (10):1495-1500
19. Schutte $\mathrm{S}$ et al (2006) A finite-element analysis model of orbital biomechanics. Vision Res 46(11):1724-1731, doi:10.1016/j. visres.2005.11.022

20. Barry JC et al (1994) Measurement of ocular alignment with photographic Purkinje I and IV reflection pattern evaluation. Invest Ophthalmol Vis Sci 35(13):4219-4235

21. von Pflugk A (1906) Beitrag zur Technik der Schieloperationen. Vornähung und Zurücknähung. In: 32nd Meeting Dtsch Ophthalmol Ges 1905 Ber Dtsch Ophthalmol Ges

22. Scott AB, Mash AJ, Jampolsky A (1975) Quantitative guidelines for exotropia surgery. Invest Ophthalmol Vis Sci 14:428-436

23. Saggau B (1976) Dosierbarkleit und Dosierungsrichtlinien von Schieloperationen in Fällen von Strabismus convergens mit oder ohne Vertikaldiffernz. University of Hamburg

24. Lang J (1981) Strabismus Operationen an den Musculi recti. Klin Mbl Augenheilk 178:271-274

25. Parks MM, Wheeler MB (1991) Concomitant esodeviations. In: Tasman W, Jaeger EA (eds) Duane's Clinical Ophthalmology. JB Lippincott Co, Philadelphia

26. Kushner BJ, Morton GV (1987) The effect of surgical technique and amount, patient age, abduction quality and deviation magnitude on surgical success rated in infantile esotropia. Binocul Vis 2(1):25-40

27. Lipton JR, Willshaw HE (1995) Prospective multicentre study of the accuracy of surgery for horizontal strabismus. Br J Ophthalmol 79(1):10-11, doi:10.1136/bjo.79.1.10

28. Mulet ME et al (2006) Adal-1 bioadhesive for sutureless recession muscle surgery: a clinical trial. Br J Ophthalmol 90(2):208-212, doi:10.1136/bjo.2005.076497

29. Jampolsky A (1975) Strabismus reoperation techniques. Trans Sect Ophthalmol Am Acad Ophthalmol Otolaryngol 79(5):704717

30. Sundaram V, Haridas A (2005) Adjustable versus non-adjustable sutures for strabismus. Cochrane Database Syst Rev 1:CD004240

31. Kushner BJ, Lucchese NJ, Morton GV (1991) Variation in axial length and anatomical landmarks in strabismic patients. Ophthalmology 98(3):400-406

32. Kushner BJ, Lucchese NJ, Morton GV (1989) The influence of axial length on the response to strabismus surgery. Arch Ophthalmol 107(11):1616-1618

33. Robinson DA (1975) A quantitative analysis of extraocular muscle cooperation and squint. Invest Ophthalmol 14(11):801-825

34. Robinson DA, O'Meara DM, Scott AB (1969) Mechanical components of human eye movements. J Appl Physiol 26:548-553 